\title{
LITERATUR
}

\section{Jahre Integrationsgeschichte im Jahrbuch der Europäischen Integration - Vom ehrgeizigen Projekt zum zuverlässigen Werkzeug}

\author{
Joachim Schild*
}

\begin{abstract}
Als Werner Weidenfeld und Wolfgang Wessels, die Herausgeber des ,Jahrbuchs der Europäischen Integration', ihr Projekt vor einem Vierteljahrhundert lancierten und 1980 das erste Exemplar vorlegten, konnten sie gewiss nicht fest mit dem Erfolg und der Dauerhaftigkeit ihres Unternehmens rechnen. Die Entwicklung der Europäischen Gemeinschaft hatte zum damaligen Zeitpunkt durch die erste Direktwahl des Europäischen Parlaments zwar einen neuen Antrieb erhalten. In ihrem Vorwort verwiesen die beiden Herausgeber damals aber auch scharfsichtig auf die „verstärkt aufbrechenden Strategiedebatten über die Entwicklungsperspektive der Europäischen Gemeinschaft"1. Und Werner Weidenfeld kam in seiner Bilanz des Standes der Integration zu Beginn der 1980er Jahre zu dem Schluss, dass ,im Blick auf die Europäische Integration nicht ohne gewichtigen Anlass die Fragezeichen im Vordergrund stehen"2. In der Tat wurde der Zustand der Europäischen Gemeinschaft zu Beginn der 1980er Jahre meist mit publizistischen Schlagwörtern wie ,Eurosklerose ' und ,Europessimismus' zu charakterisieren versucht.
\end{abstract}

Wer in dieser Entwicklungsphase der Europäischen Gemeinschaft ein Jahrbuch der Europäischen Integration auf den Markt brachte, konnte auch nicht unbedingt mit akademischen Lorbeeren rechnen. Die Zunft der Europaforscher war noch durchaus überschaubar
Werner Weidenfeld und Wolfgang Wessels (Hrsg.): Jahrbuch der Europäischen Integration 2005, Nomos: Baden-Baden 2006, ISBN 3-83291751-9; 540 Seiten, $49 €$.

Werner Weidenfeld und Wolfgang Wessels (Hrsg.): Jahrbuch der Europäischen Integration - Jahrbücher 1980 - 2002/2003, erschienen beim Europa Union Verlag, Bonn.

- ab Jahrbuch 2003/2004 erscheint das Jahrbuch bei Nomos, Baden-Baden.

Werner Weidenfeld und Wolfgang Wessels (Hrsg.): Europa von A bis Z. Taschenbuch der Europäischen Integration, 9. Auflage, Nomos: Baden-Baden 2006, ISBN 3-8329-1378-5; 498 Seiten, $19,90 €$.

und arbeitete etwas abseits vom akademischen Mainstream. Das akademische Interesse an der Europaforschung sollte dann mit der Binnenmarktverwirklichung und der damit verbundenen beschleunigten Integrationsentwicklung auch auf anderen Feldern der Integration rasch und nachhaltig zunehmen. Umso wichtiger war es, dass der eine Blüte erlebenden Europaforschung mit dem Jahrbuch ein zuverlässiges Werkzeug an die Hand gegeben wurde.

\section{Arbeitsinstrument und Nachschlagewerk}

Der Wert des Jahrbuchs als Arbeitsinstrument und Nachschlagewerk beruht nicht zuletzt auf der Existenz fester Rubriken zu den Kernins-

Prof. Dr. Joachim Schild, Lehrstuhl für Politikwissenschaft/Vergleichende Regierungslehre, Universität Trier. Werner Weidenfeld/Wolfgang Wessels (Hrsg.): Jahrbuch der Europäischen Integration 1980, Bonn 1981, S. 9. Weidenfeld/Wessels: Jahrbuch, 1980, S. 13. 
titutionen, den wichtigsten EG-/EU-Politikfeldern und auf der systematischen Berücksichtigung der Europapolitik der Mitgliedstaaten - seit 1991/92 auch der Europapolitik anderer europäischer Staaten, vor allem der Beitrittsaspiranten. Diese Entscheidung der Herausgeber, mit festen Jahrbuchrubriken zu arbeiten, erweist sich gerade im Rückblick auf ein systematisch strukturiertes Informationsangebot aus 25 Jahren als goldrichtig. Auch eine zweite Grundentscheidung hat sich als richtig erwiesen: die Entscheidung zu Gunsten einer zeitnahen Information über das Berichtsjahr. Damit haben die Herausgeber bewusst einen anderen Weg gewählt als die Herausgeber des Jahrbuchs für Internationale Politik, das bekanntlich mit einem gewissen zeitlichen Abstand auf das jeweilige Berichtsjahr zurückblickt. Das Jahrbuch für Europäische Integration hat dieser Weichenstellung gewiss eine größere Verbreitung und Wirkung über ein akademisches Zielpublikum hinaus zu verdanken.

Die Entwicklung der Jahrbuchstruktur ist ein getreuliches Abbild der funktionalen und geografischen Ausdehnung der Europäischen Gemeinschaft beziehungsweise Union. Reichten 1980 noch 11 Artikel zu den Politikbereichen der Gemeinschaft, um die wesentlichen Bereiche ihres Handelns zu erfassen, so ist die Zahl in der neuesten Ausgabe auf 16 angewachsen. Und waren 1980 zwei Artikel zur Europäischen Politischen Zusammenarbeit und $\mathrm{zu}$ den Außenbeziehungen der Europäischen Gemeinschaft zu finden, so versammelt das Kapitel Außenpolitik mittlerweile nicht weniger als 10 Artikel zu unterschiedlichen Teilbereichen und regionalen Schwerpunkten der EUAußenbeziehungen.

\section{Das Jahrbuch - eine Institution}

Dass man ein Projekt wie das Jahrbuch über eine solch lange Distanz mit Erfolg über Untiefen hinweg und an Klippen vorbei schiffen und eine durchweg hohe Qualität garantieren konnte, ist dem Geschick und dem nicht nachlassenden Engagement der Herausgeber zu verdanken. Aber was wäre ein solches Projekt ohne die Bereitschaft namhafter und vielbeschäftigter Autoren zur regelmäßigen Mitarbeit? Allein das aktuelle Jahrbuch zählt nicht weniger als 80 Autorinnen und Autoren!

Die Realisierbarkeit eines solchen Großunterfangens hängt von einer soliden institutionellen Verankerung ab. Ohne das Institut für Europäische Politik als Träger und Koordinator dieses Projekts wäre das Jahrbuch gewiss nicht entstanden und zu seinem heutigen Umfang und zu seiner publizistischen Bedeutung gelangt. Es ist somit eine Frucht der Institutionalisierung der Europaforschung in der Bundesrepublik. Aber längst ist es seinerseits zu einer festen Institution und zu einem wichtigen Bestandteil der institutionalisierten Forschungsinfrastruktur zur Europäischen Integration in Deutschland geworden.

\section{Jahre Integrationsgeschichte}

Die Herausgeber haben das Jahrbuchsjubiläum zum Anlass genommen, den Historiker Wilfried Loth (Universität Duisburg-Essen) zu bitten, einen bilanzierenden Rückblick auf die 25 Jahre Integrationsgeschichte zu werfen, die das Jahrbuch inzwischen abdeckt. In seinem gerafften Überblick über die EG-/EUEntwicklung der letzten 25 Jahre springt eine Parallele zwischen der heutigen Situation und dem Beginn der 1980er Jahre ins Auge: Nach der institutionellen Innovation der Aufwertung des Europäischen Parlaments durch seine Direktwahl, die zu vielen - zunächst enttäuschten - Hoffnungen Anlass gab, trat die Europäische Gemeinschaft in eine Phase der Unsicherheit und der Suche nach geeigneten Wegen der ,relance“ ein. Größer noch waren die Hoffnungen, die die überzeugten Europäer an die Verfassungsentwicklung knüpften, bevor auch diese infolge der französischen und niederländischen Verfassungsreferenden in Ernüchterung und Enttäuschung umschlugen. Auf Grund des in dieser Vertragsablehnung in zwei Kernländern und Gründungsmitgliedern der Europäischen Union zum Ausdruck gekommenen ,Legitimitätsverfalls“3 der Union widersteht 
Wilfried Loth in seinem Beitrag der Versuchung, die Entwicklung europäischer Integration seit 1980 als reine Erfolgsgeschichte zu beschreiben. Vielmehr nimmt er neben den wegweisenden Etappen und Erfolgen der Integrationsentwicklung auch die Defizite, Versäumnisse und unerledigten Aufgaben in den Blick, die zur aktuellen Verunsicherung und Krisensituation der Union beigetragen haben.

Gleichwohl kommt er zu einem eher optimistischen Ausblick: „Die Blockade auf dem Weg zu größerer Akzeptanz der Gemeinschaft wird sich überwinden lassen, wenn sich die Staats- und Regierungschefs darauf verständigen, die entsprechenden Bestimmungen in Teil I des Vertrags ohne die problematisch gewordenen Kontexte zur Abstimmung zu stellen. (...) Wenn die Bürger wissen, welche Zukunftsperspektive sich der jetzigen Union insgesamt bietet, werden sie souveräner darüber entscheiden können, welche Erweiterungen in ihrem Interesse sind." 4

Diesen zuversichtlichen Ausblick auf die Fähigkeit der Union, einen Weg aus ihrer Verfassungskrise zu finden und die nächsten $\mathrm{Er}$ weiterungsschritte einer zunehmend erweiterungsfeindlichen öffentlichen Meinung in vielen Mitgliedstaaten zu vermitteln, kann man teilen oder mit einigen Fragezei- chen versehen. Ob der Autor damit Recht behält und wie sich die schwierigen Jahre, die vor der Union liegen, konkret bewältigen und gestalten lassen, wird man wie immer in den letzten 25 Jahren zeitnah in den nächsten Jahrbüchern der Europäischen Integration nachlesen können.

\section{Europa von A-Z}

Werner Weidenfeld und Wolfgang Wessels hatten in ihrem Vorwort zur ersten Ausgabe des Jahrbuchs von 1980 ihre Initiative damit begründet, dass neben den durchaus vorhandenen zahlreichen Detailanalysen zur Europäischen Gemeinschaft Werke fehlten, die eher den Charakter von übersichtlichen Gesamtdarstellungen besitzen. Inzwischen gibt es auf dem deutschen wie internationalen Buchmarkt eine zunehmende Fülle von Einführungs-, Überblicksdarstellungen und Nachschlagewerken zur Europäischen Union nicht zuletzt das soeben in 9. Auflage erschienene Kompendium ,Europa von A bis Z، derselben Herausgeber. Das Jahrbuch braucht diese zunehmende ,Konkurrenz' auf Grund seines unverwechselbaren Profils aber gewiss nicht zu fürchten. Der Rezensent wünscht den aktiven Gestaltern und Trägern des Jahrbuchs auch für die nächsten 25 einen langen Atem und einen gleich bleibenden Erfolg.

3 Werner Weidenfeld/Wolfgang Wessels (Hrsg.): Jahrbuch der Europäischen Integration 2005, Baden-Baden 2006, S. 45.

4 Weidenfeld/Wessels: Jahrbuch 2005, S. 54. 